[0212-7199 (2004) 21: 12; pp 577-580] ANALES DE MEDICINA INTERNA Copyright (C) 2004 ARAN EDICIONES, S.L.

AN. MED. InTERna (Madrid) Vol. 21, N. $^{\circ} 12$, pp. $577-580,2004$

\section{Patrón de ingesta en un grupo de pacientes con síndrome de intestino irritable; relación de la ingesta de fibra con los síntomas}

\author{
R. ALLER, D. A. DE LUIS, O. IZAOLA, F. LA CALLE, L. DEL OLMO, \\ L. FERNÁNDEZ, T. ARRANZ, J. M. GONZÁLEZ HERNÁNDEZ
}

Instituto de Endocrinología y Nutrición. Unidad de Apoyo a la Investigación. Hospital Río Hortega. Facultad de Medicina. Universidad de Valladolid. Valladolid
DIETARY INTAKE OF A GROUP OF PATIENTS WITH IRRITABLE BOWEL SYNDROME; RELATION BETWEEN DIETARY FIBER AND SYMPTOMS

\section{RESUMEN}

Objetivos: El objetivo de nuestro estudio fue evaluar la ingesta de un grupo de pacientes con síndrome de intestino irritable y compararla con las ingestas internacionales recomendadas.

Pacientes y métodos: Se incluyeron un total de 53 pacientes con síndrome de intestino irritable seleccionados con un muestreo no probabilístico consecutivo. Los pacientes habían sido diagnosticados de síndrome de intestino irritable (SII) siguiendo los Criterios de Roma II. A todos los pacientes se les determinó el peso, talla, índice de masa corporal, encuesta dietética de 3 días y un test de evaluación de síntomas.

Resultados: Los 53 pacientes pesentaban una edad media de $45,67+13,6$ años y una distribución por sexo de (22 varones/31 mujeres). La ingesta calórica total fue adecuada en valores absolutos y al corregir por peso del paciente. La distribución de calorías fue un $41,5 \%$ en forma de carbohidratos, un 19,8 en proteinas y un $38,7 \%$ en lípidos, estos datos muestran un claro exceso en el consumo de grasas y defecto en el consumo de hidratos de carbono. Existió una baja ingesta de vitaminas A y D. La ingesta de vitamina B12, vitamina C y niacina fue superior que las recomendaciones internacionales. Con respecto a los minerales, existió un claro déficit de la ingesta de calcio, magnesio, yodo y zinc.

Al realizar el análisis en fibra soluble e insoluble, la primera fue inferior (fibra soluble 1,46 $\pm 0,74 \mathrm{~g} /$ día) (19\%), la fibra insoluble representó $6,21 \pm 2,67 \mathrm{~g} /$ día $(71 \%)$. La ingesta corregida de fibra por calorías fue baja 4,5 $\pm 1,2$ por cada 1.000 calorías. En el análisis de correlación sólo se correlacionó la fibra insoluble $(\mathrm{r}=-0,46$; $\mathrm{p}<0,05)$ y la fibra soluble $(\mathrm{r}=0,42 ; \mathrm{p}<0,05)$ con el factor general del sistema de puntuación y no existieron relaciones significativas con el resto de factores del sistema de puntuación.

Conclusiones: Los pacientes con síndrome de intestino irritable presentan una desviación de las ingestas de algunos minerales y vitaminas. La ingesta de fibra es pobre, existiendo una correlación entre la baja ingesta y la severidad de los síntomas generales de la enfermedad

PALABRAS CLAVE: Ingesta alimentaria. Síndrome de intestino irritable.

\section{ABSTRACT}

Objectives: The aim of our study was to evaluate the oral dietary intake of a group of patients with irritable bowel syndrome and to compate with international recommendations.

Patients and methods: A total of 53 patients with irritable bowel syndrome was enrolled in a non-propabilistic sample. Patiens were diagnose with Roma II criteria. In all patients were determined, weight, height, body mass index, dietary intake of 3 days and a symptoms scoring system.

Results: The average age of 53 patients was $45.67 \pm 13.6$ years with a distribution of sex (22 males/31 females). Caloric intakes was righ in absolut terms and corrected by weight. Distribution of calories was $41.5 \%$ of carbohidrates, a $19.8 \%$ of proteins, and a $38.7 \%$ of lipids, showing a high intake of lipids and low of carbohidrates. A low intake of vitamin $A$ and $D$ was detected. High intake of vitamin B12, vitamin $C$ and niacine was observed. A low intake of calcium, magnesium, yodo and zinc was detected.

Intake of soluble fiber was lower than insoluble fiber $(1.46 \pm 0.74$ g/day) (19\%) vs $6.21 \pm 2.67 \mathrm{~g} /$ day (71\%). Intake of fiber corrected by calories was low $4.5 \pm 1.2 \mathrm{~g} / 1000$ calories. In correlation analysis, insoluble fiber $(r=0.46 ; p<0.05)$ and soluble fiber $(r=0.42 ; p<0.05)$ were inverse correlated with general symptoms.

Conclusions: Patients with irritable bowel syndrome showed a desviation of mineral and vitamin dietary. Fiber intake is low, a correlation between fiber intake and general symptoms was detected.

Aller $R$, de Luis DA, Izaola $O$, La Calle F, del Olmo L, Fernández L, Arranz T, González. Hernández JM. Patrón de ingesta en un grupo de pacientes con síndrome de intestino irritable; relación de la ingesta de fibra con los síntomas. An Med Interna (Madrid) $2004 ; 21: 577-580$.

\section{INTRODUCCIÓN}

El síndrome de intestino irritable es una patología que se diagnostica por exclusión de patologías orgánicas del tubo digestivo, requiriéndose al menos síntomas de dolor o malestar abdominal, durante 12 semanas, con dos o tres de las siguientes características: mejoría con la defecación, inicio asociado con un cambio en la frecuencia defecatoria e inicio

Trabajo aceptado: 19 de julio de 2004

Correspondencia: Daniel Antonio de Luis. Profesor de Endocrinología y Nutrición. Director del Instituto de Endocrinología y Nutrición. Facultad de Medicina. C/ Los Perales, 16 (Urb. Las Aceñas) 47130 Simancas (Valladolid) 
asociado con un cambio en la consistencia de las heces (1). Su prevalencia es elevada, 2,3-12\%, produciendo una alta presión asistencial en las consultas de Atención Primaria y Especializada.

Se han implicado algunos factores como posibles agentes desencadenantes de la sintomatología de la enfermedad, incluyéndose alteraciones mentales, estrés en medio sociolaboral, así como alteraciones en el patrón de ingesta (2). En algunos trabajos se han sugerido la importancia no sólo de los alimentos ingeridos sino también los comportamientos alimentarios asociados con determinados alimentos (3). Otros grupos de trabajo han implicado posibles mecanismos de hipersensibilidad en tubo digestivo en la fisiopatología del síndrome de intestino irritable (4). A pesar de las diferentes hipótesis que intentan explicar este síndrome y sus claras relaciones con la ingesta de alimentos, son escasos los trabajos que estudien la ingesta de estos pacientes.

El objetivo de nuestro estudio fue evaluar la ingesta de un grupo de pacientes síndrome de intestino irritable y compararla con las ingestas internacionales recomendadas.

\section{PACIENTES Y MÉTODOS}

\section{PACIENTES}

Se incluyeron un total de 53 pacientes con síndrome de intestino irritable (SII) seleccionados con un muestreo no probabilístico consecutivo en una consulta de Digestivo del Hospital Clínico Universitario de Valladolid, desde enero 2002 a marzo 2003.

Los pacientes habían sido diagnosticados de síndrome de intestino irritable siguiendo los Criterios de Roma II (5). A todos los pacientes se les determinó el peso, talla, índice de masa corporal, encuesta dietética de 3 días y un test de síntomas.

\section{DETERMINACIONES ANTROPOMÉTRICAS}

El peso corporal fue determinado con el paciente desnudo, con una báscula calibrada en $50 \mathrm{~g}$ y la talla en un tallímetro calibrado en mm. El índice de masa corporal se calculó con la fórmula de peso $(\mathrm{kg}) /$ talla $\left(\mathrm{m}^{2}\right)$.

\section{ENCUESTA NUTRICIONAL}

Todos los pacientes recibieron instrucciones para recoger la información de sus hábitos alimentarios durante 3 días incluyendo un día de fin de semana. Las encuestas fueron revisadas por la misma dietista, introduciendo los datos en una base de datos, calibrando la dieta utilizando como tablas de referencia las de Mataix y cols. (6).

\section{SÍNTOMAS RELACIONADOS CON EL SÍNDROME DE INTESTINO IRRITABLE}

A todos los pacientes se les realizó el test (7), dividiéndose las preguntas en una escala general (náuseas, vómitos, flato, pesadez), escala intestinal (frecuencia de deposiciones diarias, defecación incompleta, uso de laxantes) y escala de dolor. Se analizaron las diferentes escalas y la escala total como suma de todas las subescalas.

\section{ANÁLISIS ESTADÍSTICO}

Los resultados se expresaron como media \pm desviación estándar y frecuencias. La distribución de las variables se analizó con el test de Kolmogorov-Smirnov.

Se realizó un estudio de correlación de Pearson para evaluar la relación existente entre las diferentes ingestas y las síntomas reacionados con el SII. Se tomó como valor de significacion estadística, un valor $\mathrm{p}<0,05$. Se realizó un análisis multivariante para determinar las variables independietes que influían sobre los síntomas.

\section{RESULTADOS}

Las características clínico epidemiológicas de los individuos con síndrome de intestino irritable se muestran en la tabla I. Como podemos comprobar se trata de un grupo de pacientes, con un índice de masa corporal dentro de la normalidad y uno de cada tres era fumador.

La tabla II muestra la ingesta media de macro- y micronutrientes comparada con las recomendaciones internacionales

\section{TABLA I}

\section{CARACTERÍSTICAS CLÍNICAS DE LOS SUJETOS A ESTUDIO (media \pm desviación estándar)}

\begin{tabular}{lc}
\hline & Síndrome de intestino irritable \\
$n$ & 53 \\
\hline Edad (años) & $45,67 \pm 13,6$ \\
Sexo (varón/mujer) & $22 / 31$ \\
IMC (kg/m²) & $25,0 \pm 73,9$ \\
Peso (kg) & $66,03 \pm 12,1$ \\
Fumadores (\%) & $30,2 \%$ \\
Alcohol & $17 \%$ \\
\hline
\end{tabular}

Alcohol: se han incluido los pacientes con una ingesta de alcohol $>30 \mathrm{~g} /$ día

(RDA) (8). La ingesta calórica total fue adecuada en valores absolutos y al corregir por peso del paciente. La distribución de calorías fue un 41,5\% en forma de carbohidratos, un 19,8\% en proteínas y un $38,7 \%$ en lípidos, estos datos muestran un claro exceso en el consumo de grasas y defecto en el consumo de hidratos de carbono.

Con referencia a las vitaminas, existió una baja ingesta de vitamina A y de vitamina $\mathrm{D}$. La ingesta de vitamina B12, vitamina $\mathrm{C}$ y niacina fue superior que las recomendaciones internacionales, el resto de vitaminas se mantuvieron en valores que oscilaron entre $80-100 \%$.

Con respecto a los minerales, existió un claro déficit de la ingesta de calcio, magnesio, yodo y zinc. Manteniéndose dentro de la normalidad la ingesta de hierro.

Realizamos un estudio específico de la ingesta de fibra, detectando una ingesta media de 7,48 \pm 3 g/día. Al realizar el análisis en fibra soluble e insoluble, la primera fue inferior (fibra soluble 1,46 $\pm 0,74 \mathrm{~g} /$ día) (19\%), la fibra insoluble representó $6,21 \pm 2,67 \mathrm{~g} /$ día $(71 \%)(\mathrm{p}<0,06)$. La ingesta corregida de fibra por calorías fue baja 4,5 $\pm 1,2 \mathrm{~g}$ por cada 1.000 calorías. 
TABLA II

PORCENTAJE DE LAS RECOMENDACIONES INTERNACIONALES (RDA) EN NUESTRA MUESTRA

\begin{tabular}{|c|c|c|}
\hline & Media (DS) & Porcentaje de RDA \\
\hline \multicolumn{3}{|l|}{ Macronutrientes: } \\
\hline Carbohidratos (g/día) & $177,17 \pm 63$ & - \\
\hline Lípidos (g/día) & $70,9 \pm 21,4$ & - \\
\hline Proteínas totales (g/day) & $81,5 \pm 26,2$ & - \\
\hline Calorías totales (cal/day) & $1646,6 \pm 496$ & - \\
\hline G de proteínas $/ \mathrm{kg}$ & $1,22 \pm 0,2$ & - \\
\hline Calorías/kg & $24,8 \pm 6,4$ & - \\
\hline \multicolumn{3}{|l|}{ Vitaminas: } \\
\hline Vit. A ( $\mu g)$ & $651 \pm 513$ & $65,1 \%$ \\
\hline Vit. D ( $\mu g)$ & $3,65 \pm 1,4$ & $36 \%$ \\
\hline Vit. C (mg) & $128,7 \pm 65$ & $214 \%$ \\
\hline Tiamina (mg) & $1,09 \pm 0,4$ & 82,5 \\
\hline Riboflavina (mg) & $1,9 \pm 0,8$ & $114,4 \%$ \\
\hline Niacina (mg) & $29,8 \pm 11,9$ & $150 \%$ \\
\hline Vit. B6 (mg) & $1,8 \pm 0,6$ & $90 \%$ \\
\hline Vit. B12 ( $\mu \mathrm{g})$ & $5,89 \pm 27$ & $292 \%$ \\
\hline Ácido fólico ( $\mu g)$ & $164,7 \pm 76$ & $89,8 \%$ \\
\hline \multicolumn{3}{|l|}{ Minerales: } \\
\hline Calcio (mg) & $763 \pm 249$ & $76,3 \%$ \\
\hline Magnesio (mg) & $222,3 \pm 78,4$ & $63,4 \%$ \\
\hline Fe mg) & $9,78 \pm 3,6$ & 97,8 \\
\hline Zinc (mg) & $8,86 \pm 4,2$ & 59,1 \\
\hline$Y(\mu g)$ & $55,6 \pm 28,8$ & $38 \%$ \\
\hline
\end{tabular}

La puntuación total de la escala de síntomas fue de 9,2 $\pm 1,9$ puntos. Al dividir esta escala en sus diferentes componentes la puntuación fue la siguiente: factor dolor 4,2 $\pm 1,2$ puntos factor intestinal 2,48 $\pm 1,1$ puntos y factor general 2,52 $\pm 0,68$ puntos. En el análisis de Pearson sólo se correlacionó la fibra insoluble de manera inversa $(r=0,46 ; \mathrm{p}<0,05)$ y la fibra soluble $(\mathrm{r}=0,42$; $\mathrm{p}<0,05)$ con el factor general el score y no existieron relaciones significativas con el resto de factores score.

Al realizar el análisis multivariante sólo permaneció en el modelo final la ingesta de fibra insoluble ajustada por la edad y el peso de los pacientes, influyendo de una manera inversa sobre la escala general de síntomas con un coeficiente de determinaron de $0,6 \mathrm{p}<0,05$.

\section{DISCUSIÓN}

Como se puede comprobar en nuestro trabajo, existen desviaciones en el patrón de ingesta de macro- y mironutrientes en nuestra muestra de pacientes con síndrome de intestino irritable. Existió una baja ingesta de hidratos de carbono, con una elevada ingesta de grasas. Esta baja ingesta de carbohidratos (verduras y frutas) en la muestra produjo una baja ingesta de fibra, en cantidades absolutas y relativas, al corregir los gramos de fibra por la ingesta calórica.

La fibra es un término que recoge una variedad de sustancias que son resistentes a la digestión de los enzimas gastroin- testinales. Habitualmente se clasifican en función de su solubilidad en el agua. Existe un grupo de fibras que se denominan fibras insolubles (celulosa, hemicelulosa, lignina), el segundo grupo son aquellas que forman un gel en presencia de agua (pectinas, gomas y mucilagos). Existen divesos trabajos que han demostrado la utilidad de la fibra en pacientes con sindrome de intestino irritable. Recientemente, Parisi y cols. (9) han demostrado en un grupo de 188 pacientes con síndrome de intestino irritable una mejoría en los síntomas al suplementar la dieta con $30 \mathrm{~g}$ de fibra en forma de salvado de trigo o con $5 \mathrm{~g}$ de goma guar parcialmente hidrolizada. El trabajo se realizó con un diseño de ensayo clínico, mejorando los dos grupos randomizados, sin existir diferencias entre ambos tratamientos. Determinados autores (10) han señalado que el perfil psicológico del paciente puede influir a la hora de predecir la respuesta a los diferentes suplementos de fibra. De este modo los pacientes con SII y con un perfil depresivo presentan mayor severidad en las escalas de dolor abdominal, los pacientes que mejor responden a los tratamientos con fibra son aquellos con menor grado de depresión antes de iniciar el tratamiento.

Como muestra nuestro trabajo existen carencias en la ingesta de determinados oligoelementos y vitaminas en este grupo de pacientes. Estos datos ya fueron comprobados por Gee y cols. (11), donde compararon la ingesta de pacientes con patología orgánica del tubo digestivo (enfermedad inflamatoria intestinal) y pacientes con patología funcional (incluyendo en este grupo pacientes con síndrome de intestino irritable) en este segundo grupo se detectó una menor ingesta de vitamina $\mathrm{A}$, vitamina $\mathrm{C}$ y ácido fólico, en nuestro trabajo también existió una disminución en la ingesta de vitamina A. En este trabajo no se analizó la ingesta de fibra. En otros trabajos (12) sólo se han detectado diferencias en la ingesta de una mayor cantidad de azúcares refinados en el grupo de pacientes con SII, no existiendo diferencias en la ingesta de fibra de estos pacientes con SII frente a pacientes sin SII. Existió una clara correlación entre la ingesta de carbohidratos refinados y fibra con los síntomas gastrointestinales y características de las heces. En otro trabajo se mostró como la ingesta de fibra derivada de vegetales era inferior en los pacientes con SII (13).

Nos podríamos preguntar a qué se debe estas alteraciones en el patrón de ingesta de micronutrientes, fibra e incluso en la alteración en la distribución porcentual de calorías en la ingesta. Probablemente pueda ser debido a alteraciones en el comportamiento alimentario secundarias a percepciones o creencias o incluso intolerancia a determinados alimentos, siendo sometidos a terapias de diversa índole incluyendo la acupuntura (14).

Nuestro trabajo confirma los hallazgos de otros grupos (15), donde se ha demostrado una relación entre la ingesta de fibra y la mejoría de los síntomas gastrointestinales sobre todo en pacientes donde alterna el estreñimiento con la urgencia fecal.

Las diferentes recomendaciones internacionales para estos pacientes y teniendo en cuenta los resultados comentados, insisten en el limitado papel de las dietas de exclusión y en la importancia de la suplementación con diferentes fibras en la dieta $(16,17)$.

En conclusión, los pacientes con síndrome de intestino irritable presentan una desviacion de las ingestas de algunos minerales y vitaminas. La ingesta de fibra es pobre, existiendo un correlación entre la baja ingesta y la severidad de los síntomas generales de la enfermedad. 


\section{Bibliografía}

1. Álvarez MA, Villagrasa M. Definición y clasificación del síndrome de intestino irritable. Med Clin (Barc) 2003; 4: 2-5.

2. Ma H, Chen Y, Liu Y. An epidemiological study on the risk factors of irritable bowel syndrome in Zhajiang, Guadong province. Zhonghua Liu Xing Bing Xue Za Zhi 2001; 22: 452-452.

3. Jarret M, Visser R, Heitkemper M. Diet triggers symptoms in women with irritable bowel syndrome. The patient's perspective. Gastroenterol Nurs 2001; 24: 246-252.

4. Zar S, Kumar D, Benson MJ. Food hypersensitivity and irritable bowel syndrome. Aliment Pharmacol Ther 2001; 15: 439-449.

5. Thompsom WG, Longsrecht GF, Drossman DA, Heaton KW, Kruis W. Functional bowel dis and functional abdominal pain. Rome II: A multinational consensus document on functional disorders. Gut 1999; 45:43-47.

6. Mataix J, Mañas M. Tablas de composición de alimentos españoles. 3a ed. Ed. University of Granada, 1998.

7. Lucey MR, Clark ML, Lowdens JO, Dawson AM. Is bran effcacious in irritable bowel syndrome? A double blind placebo controlled crossover study. Gut 1987; 28: 211-225.

8. National Research Council: Recommended Dietary Allowances, food and Nutrition Board, U.S. National Academy of Sciences. National Research Council, 1989.

9. Parisi GC, Zilli M, Miani MP, Carrara M, Bottona E, Verdianelli G. High-fiber diet supplementation in patients with irritable bowel syndrome (IBS): a multicenter, randomized, open trial comparison between wheat bran diet and partially hydrolyzed guar gum (PHGG). Dig Dis
Sci 2002; 47:1697-1704.

10. Fowlie S, Eastwood MA, Prescott R. Irritable bowel syndrome: assessment of psychological disturbance and its influence on the response to fibre supplementation. J Phsychosom Res 1992; 36: 175-180.

11. Gee MI, Grace MG, Wensel RH, Sherbaniuk RW, Thomson AB. Nutritional status of gastroenterology outpatients: comparison of inflammatory bowel disease with functional disorders. J An Diet Assoc 1985; 85 1591-1599.

12. Jarret M, Heitkemper MM, Bond EF, Georges J. Comparison of diet composition in women with or without funsctional bowel disorder. Gastroenterol Nurs 1994; 16: 253-258.

13. Hillman LC, Stace NH, Fisher Pomare EW. Dier intake and stool characteristics patients with irritable bowel syndrome. Am J Clin Nutr 1982; 36: 626-629.

14. Gabuzian KS, Sarkisian KA, Grigorian NL, Azatian ZG. Long-term results in the treatment of patients with irritable bowel syndrome. Klin Med (Mosk) 1994; 72: 47-48.

15. Lambert JP, Brunt PW, Mowat NA, Khin CC, Lai CK, Ubrrison V, Dickerson JW. The value of prescribed 'high fibre' diets for the treatment the irritable bowel syndrome. Eur J Clin Nutr 1991; 45: 12: 601-609.

16. Burden S. Dietary treatment of irritable bowel syndrome: current evidence and guidelines for future practice. J Huma Nutr Diet 2001; 14: 231-241.

17. Nagaoka Y. Dietary treatment in patients with irritable bowel syndrome. Nippon Rinsho 1992; 50: 2740-2745. 